\title{
Two solutions for the BVP of a rotating variable-thickness solid disk
}

\author{
Ashraf M. Zenkour ${ }^{1,2}$, Suzan A. Al-Ahmadi ${ }^{1}$ \\ ${ }^{1}$ Department of Mathematics, Faculty of Science, King AbdulAziz University, Jeddah, Saudi Arabia; Coresponding Author: \\ zenkour@kau.edu.sa \\ ${ }^{2}$ Department of Mathematics, Faculty of Science, Kafr El-Sheikh University, Kafr El-Sheikh, Egypt; zenkour@sci.kfs.edu.eg
}

Received 23 November 2010; revised 25 December 2010; accepted 28 December 2010.

\begin{abstract}
This paper presents the analytical and numerical solutions for a rotating variable-thickness solid disk. The outer edge of the solid disk is considered to have free boundary conditions. The governing equation is derived from the basic equations of the rotating solid disk and it is solved analytically or numerically using finite difference algorithm. Both analytical and numerical results for the distributions of stress function and stresses of variable-thickness solid disks are obtained. Finally, the distributions of stress function and stresses are presented and the appropriate comparisons and discussions are made at the same angular velocity.
\end{abstract}

Keywords: Rotating, Solid Disk, Variable Thickness, Analytical Method, Finite Difference Method

\section{INTRODUCTION}

The theoretical and experimental investigations on the rotating solid disks have been widespread attention due to the great practical importance in mechanical engineering. Rotating disks have received a great deal of attention because of their widely used in many mechanical and electronic devices. They have extensive practical engineering application such as in steam and gas turbines, turbo generators, flywheel of internal combustion engines, turbojet engines, reciprocating engines, centrifugal compressors and brake disks. The problems of rotating solid disks have been performed under various interesting assumptions and the topic can be easily found in most of the standard elasticity books [1,2]. For a better utilization of the material, it is necessary to allow variation of the effective material or thickness properties in one direction of the solid disk.

The problems of rotating variable-thickness solid disks are rare in the literature. Most of the research works are concentrated on the analytical solutions of rotating isotropic disks with simple cross-section geometries of uniform thickness and specifically variable thickness. The solution of a rotating solid disk with constant thickness is obtained by Gamer $[3,4]$ taking into account the linear strain hardening material behavior. The inelastic and viscoelastic deformations of rotating variablethickness solid disks have been presented in the literature [5-8]. Eraslan [5], and Eraslan and Orcan [6] have analytically studied rotating disks of exponentially varying thickness and of linearly strain hardening material. Eraslan [7] has presented the stress distributions in elastic-plastic rotating disks with elliptical thickness profiles using Tresca and von Mises criteria. Zenkour and Allam [8] have developed analytical solution for the analysis of deformation and stresses in elastic rotating viscoelastic solid and annular disks with arbitrary crosssections of continuously variable thickness.

As many rotating components in use have complex cross-sectional geometries, they cannot be dealt with using the existing analytical methods. Numerical methods, such as the finite element method [9], the boundary element method [10] and Runge-Kutta's algorithm [11], can be applied to cope with these rotating components. You et al. [11] have numerically studied rotating solid disks of uniform thickness and constant density as well as annular disks of variable thickness and variable density. In a recent paper, Zenkour and Mashat [12] have presented both analytical and numerical solutions for the analysis of deformation and stresses in elastic rotating disks with arbitrary cross-sections of continuously variable thickness.

In this article, a unified governing equation is firstly derived from the basic equations of the rotating variablethickness solid disk and the proposed stress-strain relationship. The analytical solution for rotating solid disk with arbitrary cross-section of continuously variable thickness is presented. Next, the finite difference method 
(FDM) is also introduced to solve the governing equation. A comparison between both analytical and numerical solutions is made. Finally, a number of application examples are given to demonstrate the validity of the proposed method.

\section{BASIC EQUATIONS}

As the effect of thickness variation of rotating solid disks can be taken into account in their equation of motion, the theory of the variable-thickness solid disks can give good results as that of uniform-thickness disks as long as they meet the assumption of plane stress. The present solid disk is considered as a single layer of variable thickness. After considering this effect, the equation of motion of rotating disks with variable thickness can be written as

$$
\frac{\mathrm{d}}{\mathrm{d} r}\left(h r \sigma_{r}\right)-h \sigma_{\theta}+h \rho \omega^{2} r^{2}=0,
$$

where $\sigma_{r}$ and $\sigma_{\theta}$ are the radial and circumferential stresses, $h$ is the variable thickness of the disk, $r$ is the radial coordinate, $\rho$ is the material density of the rotating solid disk and $\omega$ is the constant angular velocity.

The relations between the radial displacement $u$ and the strain components are irrespective of the thickness of the rotating solid disk. They can be written as

$$
\varepsilon_{r}=\frac{\mathrm{d} u}{\mathrm{~d} r}, \quad \varepsilon_{\theta}=\frac{u}{r},
$$

where $\varepsilon_{r}$ and $\varepsilon_{\theta}$ are the radial and circumferential strains, respectively. The above geometric relations lead to the following condition of deformation harmony:

$$
\frac{\mathrm{d}}{\mathrm{d} r}\left(r \varepsilon_{\theta}\right)-\varepsilon_{r}=0
$$

For the elastic deformation, the constitutive equations for the variable-thickness solid disk can be described with Hooke's law

$$
\varepsilon_{r}=\frac{\sigma_{r}-v \sigma_{\theta}}{E}, \varepsilon_{\theta}=\frac{\sigma_{\theta}-v \sigma_{r}}{E},
$$

where $E$ is Young's modulus and $v$ is Poisson's ratio. Introducing the stress function $\varphi$ and assuming that the following relations hold between the stresses and the stress function

$$
\sigma_{r}=\frac{\varphi}{h r}, \sigma_{\theta}=\frac{1}{h} \frac{\mathrm{d} \varphi}{\mathrm{d} r}+\rho \omega^{2} r^{2} .
$$

Substituting Eq.5 into Eq.4, one obtains

$$
\begin{aligned}
& \varepsilon_{r}=\frac{1}{E}\left[\left(\frac{1}{h r}-\frac{v}{h} \frac{\mathrm{d}}{\mathrm{d} r}\right) \varphi-v \rho \omega^{2} r^{2}\right], \\
& \varepsilon_{\theta}=\frac{1}{E}\left[\left(\frac{1}{h} \frac{\mathrm{d}}{\mathrm{d} r}-\frac{v}{h r}\right) \varphi+\rho \omega^{2} r^{2}\right] .
\end{aligned}
$$

\section{FORMULATION AND ANALYTIC ELASTIC SOLUTION}

The substitution of Eq.6 into Eq.3 produces the following confluent hypergeometric differential equation for the stress function $\varphi(r)$ :

$$
\begin{aligned}
& r^{2} \frac{\mathrm{d}^{2} \varphi}{\mathrm{d} r^{2}}+r\left(1-\frac{r}{h} \frac{\mathrm{d} h}{\mathrm{~d} r}\right) \frac{\mathrm{d} \varphi}{\mathrm{d} r} \\
& -\left(1-\frac{v r}{h} \frac{\mathrm{d} h}{\mathrm{~d} r}\right) \varphi+(3+v) h \rho \omega^{2} r^{3}=0 .
\end{aligned}
$$

The boundary conditions for the rotating solid disk are

$$
\begin{array}{llll}
\sigma_{r}=\sigma_{\theta} & \text { at } & r & =0, \\
\sigma_{r}=0 & \text { at } & r & =b .
\end{array}
$$

The thickness of the solid disk is assumed to vary nonlinearly through the radial direction. It is assumed to be in terms of a simple exponential power law distribution according to the following case:

$$
h(r)=h_{0} \mathrm{e}^{-n\left(\frac{r}{b}\right)^{k}},
$$

where $h_{0}$ is the thickness at the middle of the disk, $n$ and $k$ are geometric parameters and $b$ is the outer radius of the disk (see Figure 1). The value of $n$ equal to zero represents a uniform-thickness solid disk while the value of $k$ equal to unity represents a linearly decreasing variable-thickness solid disk. For small $k$ and large $n(k=0.7$ or 1.5 and $n=2)$ the profile of the solid disk is concave while it is convex for large $k$ and small $n(k=2.5$ and $n=$ $0.5)$. It is to be noted that the parameter $n$ determines the thickness at the outer edge of the solid disk relative to $h_{0}$ while the parameter $k$ determine the shape of the profile.

Introducing the following dimensionless forms:

$$
\begin{aligned}
& R=r / b \\
& \Omega=\omega b \sqrt{\rho(3+v)}, \\
& \Phi(R)=\frac{1}{b h_{0} \Omega^{2}} \varphi(r), \\
& \left(\varepsilon_{1}, \varepsilon_{2}\right)=\frac{E}{\Omega^{2}}\left(\varepsilon_{r}, \varepsilon_{\theta}\right), \\
& \left(\sigma_{1}, \sigma_{2}\right)=\frac{1}{\Omega^{2}}\left(\sigma_{r}, \sigma_{\theta}\right) .
\end{aligned}
$$

Then, Eq.7 may be written in the following simple form

$R^{2} \frac{\mathrm{d}^{2} \Phi}{\mathrm{d} R^{2}}+\left(1+k n R^{k}\right) R \frac{\mathrm{d} \Phi}{\mathrm{d} R}-\left(1+k n v R^{k}\right) \Phi+\mathrm{e}^{-n R^{k}} R^{3}=0$.

The general solution of the above equation can be 


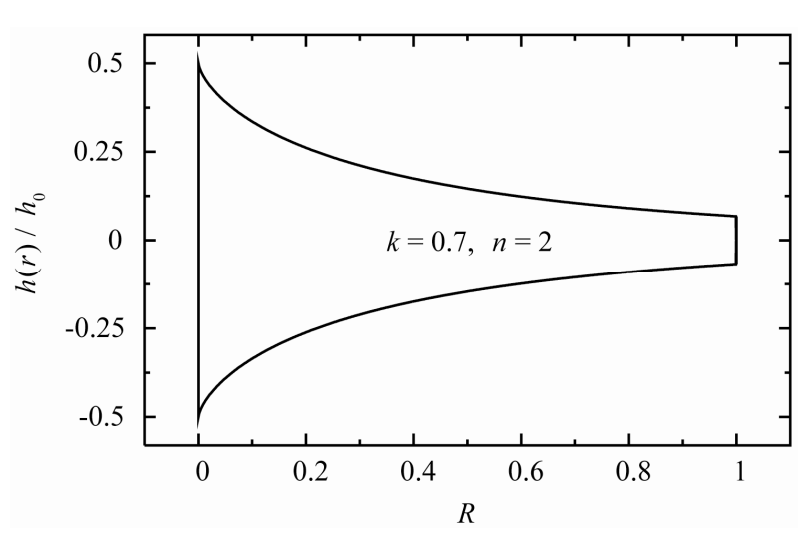

(a)

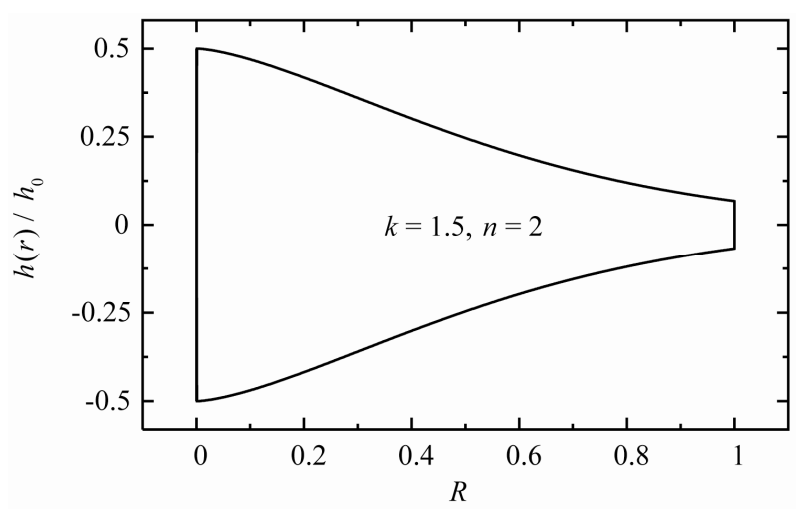

(b)

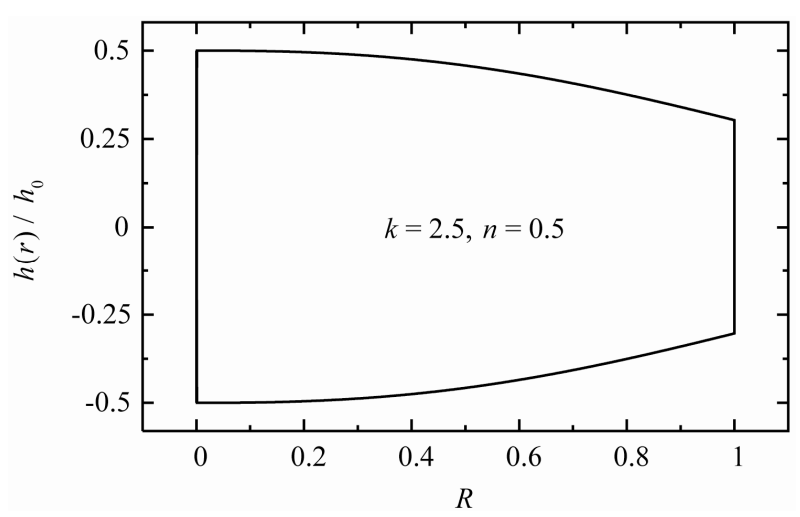

(c)

Figure 1. Variable-thickness solid disk profiles for (a) $k=0.7$ and $n=2$, (b) $k=1.5$ and $n=2$ and (c) $k=2.5$ and $n=0.5$.

written as

$$
\begin{aligned}
\Phi(R)= & R^{-\frac{k}{2}} \mathrm{e}^{-\frac{n}{2} R^{k}}\left\{M_{i, j}(R)\left[C_{1}-\int^{R} F(\xi) W_{i, j}(\xi) \mathrm{d} \xi\right]\right. \\
& \left.+W_{i, j}(R)\left[C_{2}+\int^{R} F(\xi) M_{i, j}(\xi) \mathrm{d} \xi\right]\right\},
\end{aligned}
$$

where $C_{1}$ and $C_{2}$ are arbitrary constants, $\xi$ is a dummy parameter, $M_{i, j}$ and $W_{i, j}$ are Whittaker's functions

$$
M_{i, j}(R)=M\left(i, j, n R^{k}\right), \quad W_{i, j}(R)=W\left(i, j, n R^{k}\right),
$$

in which

$$
i=\frac{1}{2}-\frac{v}{k}, \quad j=\frac{1}{k}, \quad k>0 .
$$

In addition, the function $F(R)$ is given in terms of Whittaker's functions by

$$
F(R)=\frac{R^{\frac{k}{2}+2} \mathrm{e}^{-\frac{n}{2} R^{k}}}{(1-v) W_{i, j}(R) M_{i+1, j}(R)+k M_{i, j}(R) W_{i+1, j}(R)} .
$$

The substitution of Eq.12 into Eq.5 with the aid of the dimensionless forms given in Eq.10 gives the radial and circumferential stresses in the following forms:

$$
\begin{gathered}
\sigma_{1}(R)=R^{-\frac{k}{2}-1} \mathrm{e}^{\frac{n}{2} R^{k}}\left\{M_{i, j}(R)\left[C_{1}-\int^{R} F(\xi) W_{i, j}(\xi) \mathrm{d} \xi\right]\right. \\
\left.+W_{i, j}(R)\left[C_{2}+\int^{R} F(\xi) M_{i, j}(\xi) \mathrm{d} \xi\right]\right\}, \\
\sigma_{2}(R)=\mathrm{e}^{-n R^{k}} \frac{\mathrm{d} \Phi}{\mathrm{d} R}+\frac{R^{2}}{3+v} .
\end{gathered}
$$

Here, the first derivative of the stress function $\Phi(R)$ with respect to $R$ may be given easily by using Eq.12. Note that the first derivatives of Whittaker's functions $M_{i, j}$ and $W_{i, j}$ can be represented by

$$
\begin{aligned}
\frac{\mathrm{d}}{\mathrm{d} R} M_{i, j}(R)= & \frac{k}{R}\left[\left(\frac{1}{2} n R^{k}-i\right) M_{i, j}(R)\right. \\
& \left.+\left(\frac{1}{2}+i+j\right) M_{i+1, j}(R)\right], \\
\frac{\mathrm{d}}{\mathrm{d} R} W_{i, j}(R)= & \frac{k}{R}\left[\left(\frac{1}{2} n R^{k}-i\right) W_{i, j}(R)-W_{i+1, j}(R)\right] .
\end{aligned}
$$

Finally, the stress function $\Phi(R)$ and consequently the stresses $\sigma_{1}(R)$ and $\sigma_{2}(R)$ may be determined completely after applied the dimensionless of the boundary conditions given in Eq.8.

\section{FINITE DIFFERENCE ALGORITHM}

The resolution of the elastic problem of rotating solid disk with variable thickness is to solve a second-order differential equation, Eq.11, under the given boundary conditions $\Phi(0)=\Phi(1)=0$ such that $\sigma_{1}(0)=\sigma_{2}(0)$. Eq.11 can be written in the following general form:

$$
\Phi^{\prime \prime}=p(R) \Phi^{\prime}+q(R) \Phi+s(R),
$$

where the prime $(')$ denotes differentiation with respect to $R$ and 


$$
\begin{aligned}
& p(R)=-\frac{1+k n R^{k}}{R}, \\
& q(R)=\frac{1+k n v R^{k}}{R^{2}}, \\
& s(R)=-\mathrm{e}^{-n R^{k}} R .
\end{aligned}
$$

It is clear that the above problem has a unique solution because $p(R), q(R)$, and $s(R)$ are continuous on $[0,1]$ and $q(R)>0$ on $[0,1]$. The linear second-order boundary value problem given in Eq.19 requires that difference-quotient approximations be used for approximating $\Phi^{\prime}$ and $\Phi^{\prime \prime}$. First we select an integer $N>0$ and divided the interval $[0,1]$ into $(N+1)$ equal subintervals, whose end points are the mesh points $R_{i}=i \Delta R$, for $i=0,1, \ldots, N+1$, where $\Delta R=1 /(N+1)$. At the interior mesh points, $R_{i}, i=1,2, \ldots, N$, the differential equation to the approximated is

$$
\Phi^{\prime \prime}\left(R_{i}\right)=p\left(R_{i}\right) \Phi^{\prime}\left(R_{i}\right)+q\left(R_{i}\right) \Phi\left(R_{i}\right)+s\left(R_{i}\right) .
$$

If we apply the centered difference approximations of $\Phi^{\prime}\left(R_{i}\right)$ and $\Phi^{\prime \prime}\left(R_{i}\right)$ to Eq.21, we arrive at the system (see Eq.22):

for each $i=1,2, \ldots, N$. The $N$ equations, together with the boundary conditions

$$
\begin{gathered}
\Phi_{0}=0, \\
\Phi_{N+1}=0,
\end{gathered}
$$

are sufficient to determine the unknowns $\Phi_{i}$, $i=0,1,2, \ldots, N+1$. The resulting system of Eq.22 is expresses in the tri-diagonal $N \times N$-matrix form:

$$
A \Phi=B,
$$

where

$$
\begin{aligned}
& A_{i, i}=-2-(\Delta R)^{2} q\left(R_{i}\right), \quad i=1,2, \ldots, N, \\
& A_{i, i+1}=1-\frac{\Delta R}{2} p\left(R_{i}\right), \quad i=1,2, \ldots, N-1, \\
& A_{i, i-1}=1+\frac{\Delta R}{2} p\left(R_{i}\right), \quad i=2,3, \ldots, N, \\
& A_{i, j}=A_{j, i}=0, \quad i=1,2, \ldots, N-2, j=3,4, \ldots, N, \quad j \geq i+2, \\
& B_{i}=(\Delta R)^{2} s\left(R_{i}\right), \quad i=1,2, \ldots, N .
\end{aligned}
$$

The solution of the finite difference discretization of the two-point linear boundary value problem can therefore be found easily even for very small mesh sizes.

\section{NUMERICAL EXAMPLES AND DISCUSSION}

Some numerical examples for the rotating variablethickness solid disks will be given according the analytical and numerical solutions $(v=0.3)$. According to Eq.10, the stress function $\Phi$, the radial stress $\sigma_{1}$ and the circumferential stress $\sigma_{2}$ determined as per the analytical solution are compared with those obtained by the numerical FDM solution.

The results of the present investigations for the stress function $\Phi$ are reported in Table 1 for rotating variable-thickness solid disk with $k=2.5$ and $n=0.5$. For this example, $N=9,19,39$ and 79, so $\Delta R$ has the corresponding values $0.1,0.05,0.025$ and 0.0125 , respectively. The FDM gives results compared well with the exact solution, especially for small values of $\Delta R$. The relative error between the exact method and the FDM with $\Delta R=0.0125$, may be less than $1.3 \times 10^{-4}$.

Richardson's extrapolation method is applied here with $\Delta R=0.1,0.05,0.025$, and 0.0125 and the obtained results are listed in Table 2. These extrapolations are given, respectively by

$$
\begin{gathered}
\operatorname{Ext}_{1 i}=\frac{4 \Phi_{i}(\Delta R=0.05)-\Phi_{i}(\Delta R=0.1)}{3}, \\
\operatorname{Ext}_{2 i}=\frac{4 \Phi_{i}(\Delta R=0.025)-\Phi_{i}(\Delta R=0.05)}{3}, \\
\operatorname{Ext}_{3 i}=\frac{4 \Phi_{i}(\Delta R=0.0125)-\Phi_{i}(\Delta R=0.025)}{3}, \\
\operatorname{Ext}_{4 i}=\frac{16 \mathrm{Ext}_{2 i}-\mathrm{Ext}_{1 i}}{15},
\end{gathered}
$$

Table 2 shows that all extrapolations results are correct to the decimal places listed. In fact, if sufficient digits are maintained, the approximation of $\mathrm{Ext}_{4 i}$ gives results those agree with the exact solution with maximum difference error of $1.0 \times 10^{-9}$ at some of the mesh points. Additional results for the stress function $\Phi$ are reported for rotating variable-thickness solid disk with $k$ $=0.7$ and $n=2$ in Table 3 and with $k=1.5$ and $n=2$ in Table 4. Once again, the FDM gives results compared well with the exact analytical solution, especially for small values of $\Delta R$.

Now the least square method and curve fitting are used for the discrete results of the stress function $\Phi$. So, one can get easily the radial and circumferential stresses

$$
-\left[1+\frac{\Delta R}{2} p\left(R_{i}\right)\right] \Phi_{i-1}+\left[2+(\Delta R)^{2} q\left(R_{i}\right)\right] \Phi_{i}-\left[1-\frac{\Delta R}{2} p\left(R_{i}\right)\right] \Phi_{i+1}=-(\Delta R)^{2} s\left(R_{i}\right)
$$


Table 1. Dimensionless stress function $\Phi$ of a rotating variable-thickness solid disk $(k=2.5, n=0.5)$.

\begin{tabular}{|c|c|c|c|c|c|}
\hline \multirow{2}{*}{$R_{i}$} & \multicolumn{4}{|l|}{ FDM } & \multirow{2}{*}{ Analytical } \\
\hline & $\Delta R=0.1$ & $\Delta R=0.05$ & $\Delta R=0.025$ & $\Delta R=0.0125$ & \\
\hline 0 & 0 & 0 & 0 & 0 & 0 \\
\hline 0.0125 & --- & --- & --- & 0.001317819 & 0.001318247 \\
\hline 0.0250 & --- & --- & 0.002632275 & 0.002634312 & 0.002635012 \\
\hline 0.0375 & --- & --- & --- & 0.003947918 & 0.003948803 \\
\hline 0.0500 & --- & 0.005341761 & 0.005253982 & 0.005270818 & 0.005258119 \\
\hline 0.0625 & --- & --- & --- & 0.006560297 & 0.006561453 \\
\hline 0.0750 & --- & --- & 0.007852303 & 0.007856045 & 0.007857295 \\
\hline 0.0875 & --- & --- & --- & 0.009142869 & 0.009144132 \\
\hline 0.1000 & 0.010333909 & 0.010398523 & 0.010414946 & 0.010419072 & 0.010420449 \\
\hline 0.1125 & --- & --- & --- & 0.011683316 & 0.011684731 \\
\hline 0.1250 & --- & --- & 0.012929711 & 0.012934026 & 0.012935466 \\
\hline 0.1375 & --- & --- & --- & 0.014169696 & 0.014171142 \\
\hline 0.1500 & --- & 0.015367662 & 0.015384444 & 0.015388799 & 0.015390252 \\
\hline 0.1625 & --- & --- & --- & 0.016589850 & 0.016591293 \\
\hline 0.1750 & --- & --- & 0.017767073 & 0.017771346 & 0.017772771 \\
\hline 0.1875 & --- & --- & --- & 0.018931799 & 0.018933196 \\
\hline 0.2000 & 0.019984044 & 0.020049282 & 0.020065635 & 0.020069727 & 0.020071091 \\
\hline 0.2125 & --- & --- & --- & 0.021183663 & 0.021184986 \\
\hline 0.2250 & --- & --- & 0.022268320 & 0.022272149 & 0.022273424 \\
\hline 0.2375 & --- & --- & --- & 0.023333741 & 0.023334964 \\
\hline 0.2500 & --- & 0.024349525 & 0.024363514 & 0.024367011 & 0.024368176 \\
\hline 0.2625 & --- & --- & --- & 0.025370546 & 0.025371649 \\
\hline 0.2750 & --- & --- & 0.026339842 & 0.026342953 & 0.026343989 \\
\hline 0.2875 & --- & --- & --- & 0.027282855 & 0.027283820 \\
\hline 0.3000 & 0.028132394 & 0.028175489 & 0.028186218 & 0.028188899 & 0.028189791 \\
\hline 0.3125 & --- & --- & --- & 0.029059753 & 0.029060568 \\
\hline 0.3250 & --- & --- & 0.029891894 & 0.029894109 & 0.029894846 \\
\hline 0.3375 & --- & --- & --- & 0.030690687 & 0.030691343 \\
\hline 0.3500 & --- & 0.031439588 & 0.031446504 & 0.031448230 & 0.031448803 \\
\hline 0.3625 & --- & --- & --- & 0.032165512 & 0.032166002 \\
\hline 0.3750 & --- & --- & 0.032840118 & 0.032841338 & 0.032841742 \\
\hline 0.3875 & --- & --- & --- & 0.033474542 & 0.033474861 \\
\hline 0.4000 & 0.034048816 & 0.034060453 & 0.034063288 & 0.034063994 & 0.034064227 \\
\hline 0.4125 & --- & --- & --- & 0.034608596 & 0.034608743 \\
\hline 0.4250 & --- & --- & 0.035107098 & 0.035107288 & 0.035107349 \\
\hline 0.4375 & --- & --- & --- & 0.035559047 & 0.035559023 \\
\hline 0.4500 & --- & 0.035964469 & 0.035963207 & 0.035962888 & 0.035962780 \\
\hline 0.4625 & --- & --- & --- & 0.036317868 & 0.036317677 \\
\hline 0.4750 & --- & --- & 0.036623899 & 0.036623085 & 0.036622811 \\
\hline 0.4875 & --- & --- & --- & 0.036877677 & 0.036877324 \\
\hline 0.5000 & 0.037107585 & 0.037087271 & 0.037082122 & 0.037080832 & 0.037080400 \\
\hline 0.5125 & --- & --- & --- & 0.037231777 & 0.037231269 \\
\hline 0.5250 & --- & --- & 0.037331532 & 0.037329791 & 0.037329209 \\
\hline 0.5375 & --- & --- & --- & 0.037374198 & 0.037373544 \\
\hline 0.5500 & --- & 0.037375156 & 0.037366529 & 0.037364369 & 0.037363648 \\
\hline 0.5625 & --- & --- & --- & 0.037299729 & 0.037298942 \\
\hline 0.5750 & --- & --- & 0.037182291 & 0.037179570 & 0.037178902 \\
\hline 0.5875 & --- & --- & --- & 0.037003958 & 0.037003051 \\
\hline
\end{tabular}




\begin{tabular}{|c|c|c|c|c|c|}
\hline 0.6000 & 0.036832186 & 0.036786324 & 0.036774810 & 0.036771929 & 0.036770967 \\
\hline 0.6125 & --- & --- & --- & 0.036483294 & 0.036482282 \\
\hline 0.6250 & --- & --- & 0.036140911 & 0.036137738 & 0.036136679 \\
\hline 0.6375 & --- & --- & --- & 0.035734998 & 0.035733896 \\
\hline 0.6500 & --- & 0.035291940 & 0.035278284 & 0.035274868 & 0.035273729 \\
\hline 0.6625 & --- & --- & --- & 0.034757198 & 0.034756025 \\
\hline 0.6750 & --- & --- & 0.034185494 & 0.034181892 & 0.034180690 \\
\hline 0.6875 & --- & --- & --- & 0.033548911 & 0.033547685 \\
\hline 0.7000 & 0.032936503 & 0.032876919 & 0.032862001 & 0.032858271 & 0.032857027 \\
\hline 0.7125 & --- & --- & --- & 0.032110047 & 0.032108789 \\
\hline 0.7250 & --- & --- & 0.031308165 & 0.031304368 & 0.031303102 \\
\hline 0.7375 & --- & --- & --- & 0.030441421 & 0.030440152 \\
\hline 0.7500 & --- & 0.029540441 & 0.029525248 & 0.029521449 & 0.029520183 \\
\hline 0.7625 & --- & --- & --- & 0.028544751 & 0.028543493 \\
\hline 0.7750 & --- & --- & 0.027515416 & 0.027511682 & 0.027510437 \\
\hline 0.7875 & --- & --- & --- & 0.026422653 & 0.026421427 \\
\hline 0.8000 & 0.025353741 & 0.025296129 & 0.025281728 & 0.025278128 & 0.025276927 \\
\hline 0.8125 & --- & --- & --- & 0.024078628 & 0.024077458 \\
\hline 0.8250 & --- & --- & 0.022828123 & 0.022824727 & 0.022823594 \\
\hline 0.8375 & --- & --- & --- & 0.021517051 & 0.021515961 \\
\hline 0.8500 & --- & 0.020171897 & 0.020159404 & 0.020156281 & 0.020155240 \\
\hline 0.8625 & --- & --- & --- & 0.018743148 & 0.018742161 \\
\hline 0.8750 & --- & --- & 0.017281209 & 0.017278432 & 0.017277507 \\
\hline 0.8875 & --- & --- & --- & 0.015762966 & 0.015762106 \\
\hline 0.9000 & 0.014247568 & 0.014209433 & 0.014199988 & 0.014197627 & 0.014196841 \\
\hline 0.9125 & --- & --- & --- & 0.012583344 & 0.012582635 \\
\hline 0.9250 & --- & --- & 0.010922960 & 0.010921087 & 0.010920462 \\
\hline 0.9375 & --- & --- & --- & 0.009211874 & 0.009211340 \\
\hline 0.9500 & --- & 0.007463350 & 0.007458083 & 0.007456766 & 0.007456327 \\
\hline 0.9625 & --- & --- & --- & 0.005656864 & 0.005656526 \\
\hline 0.9750 & --- & --- & 0.003814000 & 0.003813309 & 0.003813078 \\
\hline 0.9875 & --- & --- & --- & 0.001927282 & 0.001927164 \\
\hline 1.0000 & 0 & 0 & 0 & 0 & 0 \\
\hline
\end{tabular}

Table 2. Dimensionless stress function $\Phi$ of a rotating variable-thickness solid disk using Richardson's extrapolation method with different values of $\Delta R(k=2.5, n=0.5)$.

\begin{tabular}{llllll}
\hline$R_{i}$ & Ext $_{1 i}$ & Ext $_{2 i}$ & Ext $_{3 i}$ & Ext $_{4 i}$ & Analytical \\
\hline 0.0 & 0 & 0 & 0 & 0 & 0 \\
0.1 & 0.010420061 & 0.010420421 & 0.010420447 & 0.010420444 & 0.010420449 \\
0.2 & 0.020071028 & 0.020071086 & 0.020071091 & 0.020071090 & 0.020071091 \\
0.3 & 0.028189855 & 0.028189794 & 0.028189792 & 0.028189790 & 0.028189791 \\
0.4 & 0.034064332 & 0.034064233 & 0.034064229 & 0.034064227 & 0.034064227 \\
0.5 & 0.037080500 & 0.037080406 & 0.037080401 & 0.037080400 & 0.037080400 \\
0.6 & 0.036771037 & 0.036770972 & 0.036770969 & 0.036770967 & 0.036770967 \\
0.7 & 0.032857058 & 0.032857029 & 0.032857028 & 0.032857028 & 0.032857027 \\
0.8 & 0.025276926 & 0.025276927 & 0.025276928 & 0.025276927 & 0.025276927 \\
0.9 & 0.014196825 & 0.014196839 & 0.014196840 & 0.014196840 & 0.014196841 \\
1.0 & 0 & 0 & 0 & 0 & 0 \\
\hline
\end{tabular}


Table 3. Dimensionless stress function $\Phi$ of a rotating variable-thickness solid disk $(k=0.7, n=2)$.

\begin{tabular}{llllll}
\hline \multirow{2}{*}{$R_{i}$} & FDM & & & & \\
\cline { 2 - 4 } & $\Delta R=0.1$ & $\Delta R=0.05$ & $\Delta R=0.025$ & $\Delta R=0.0125$ & 0 \\
\hline 0.0 & 0 & 0 & 0 & 0 & 0.003891568 \\
0.1 & 0.003893631 & 0.003892229 & 0.003891774 & 0.003891627 & 0.006897877 \\
0.2 & 0.006913732 & 0.006901872 & 0.006898890 & 0.006898133 & 0.008971625 \\
0.3 & 0.008996897 & 0.008977938 & 0.008973209 & 0.008972022 & 0.010102762 \\
0.4 & 0.010132490 & 0.010110178 & 0.010104619 & 0.010103227 & 0.010321685 \\
0.5 & 0.010351793 & 0.010329193 & 0.010323563 & 0.010322155 & 0.009683519 \\
0.6 & 0.009710919 & 0.009690352 & 0.009685228 & 0.009683947 & 0.008256930 \\
0.7 & 0.008279371 & 0.008262527 & 0.008258329 & 0.008257280 & 0.006116796 \\
0.8 & 0.006132695 & 0.006120761 & 0.006117787 & 0.006117044 & 0.003339509 \\
1.0 & 0.003347797 & 0.003341577 & 0.003340026 & 0.003339638 & 0 \\
\hline
\end{tabular}

Table 4. Dimensionless stress function $\Phi$ of a rotating variable-thickness solid disk $(k=1.5, n=2)$.

\begin{tabular}{llllll}
\hline \multirow{2}{*}{$R_{i}$} & FDM & & & & \\
\cline { 2 - 4 } & $\Delta R=0.1$ & $\Delta R=0.05$ & $\Delta R=0.025$ & $\Delta R=0.0125$ & 0 \\
\hline 0.0 & 0 & 0 & 0 & 0 & 0.005416705 \\
0.1 & 0.005412300 & 0.005415269 & 0.005416315 & 0.005416605 & 0.009932747 \\
0.2 & 0.009985706 & 0.009945759 & 0.009935982 & 0.009933554 & 0.013033300 \\
0.3 & 0.013135277 & 0.013058534 & 0.013039590 & 0.013034871 & 0.014512487 \\
0.4 & 0.014641868 & 0.014544543 & 0.014520482 & 0.014514484 & 0.014415043 \\
0.5 & 0.014549332 & 0.014448330 & 0.014423346 & 0.014417117 & 0.012959495 \\
0.6 & 0.013080378 & 0.012989467 & 0.012966973 & 0.012961364 & 0.010460010 \\
0.7 & 0.010555201 & 0.010483617 & 0.010465900 & 0.010461482 & 0.007259771 \\
0.9 & 0.007323059 & 0.007275470 & 0.007263688 & 0.007260750 & 0.003682061 \\
\hline
\end{tabular}

since we have $\Phi$ as a continuous function of $R$. The distributions of the stress function, radial and circumferential stresses are presented in Figure 2. The numerical FDM solution is compared with the exact analytical solution for the rotating variable-thickness solid disk with $k=2.5$ and $n=0.5$. It can be seen that the FDM can describe the stress function and stresses through the thickness of the rotating solid disk very well enough.

For the sake of completeness and accuracy, additional results for the stress function and stresses are presented in Figures 3-5 for different values of the geometric parameters $k$ and $n$. Figure 3 shows the stress function $\Phi$ through the radial direction of the rotating solid disk with $k=2.5, n=0.5 ; k=0.7, n=2$ and $k=1.5, n=2$. Similar results for the radial $\sigma_{1}$ and the circumferential $\sigma_{2}$ stresses are plotted in Figures 4 and 5. Figure 3 shows that the stress function $\Phi$ increases as $k$ increases and this irrespective of the value of $n$ (see also Tables 3 and 4). Figures 4 and 5 show that $k=2.5, n=$ 0.5 gives the largest stresses. The intersection of the two cases $k=0.7, n=2$ and $k=1.5, n=2$ may be occurred at $R=0.1$ for the radial stress and at $R=0.15$ for the circumferential stress.

It is clear that, the FDM gives stress function and, 


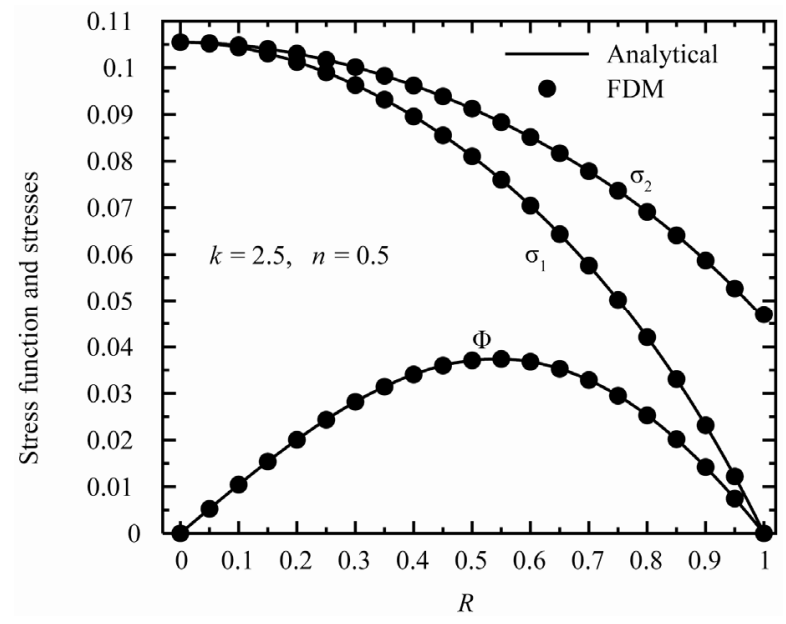

Figure 2. Stress function $\Phi$, radial stress $\sigma_{1}$ and circumferential stress $\sigma_{2}$ for the variable-thickness solid disk.

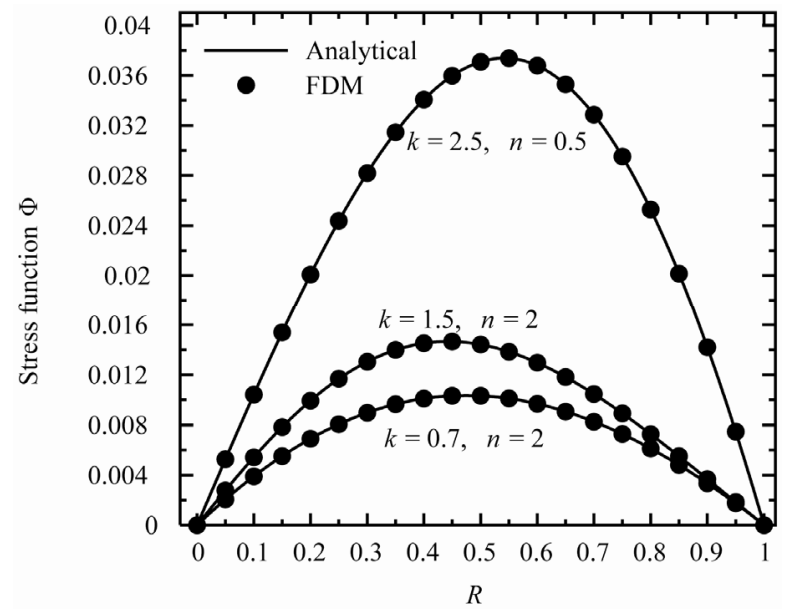

Figure 3. Stress function $\Phi$ of the variable-thickness solid disk for different values of $k$ and $n$.

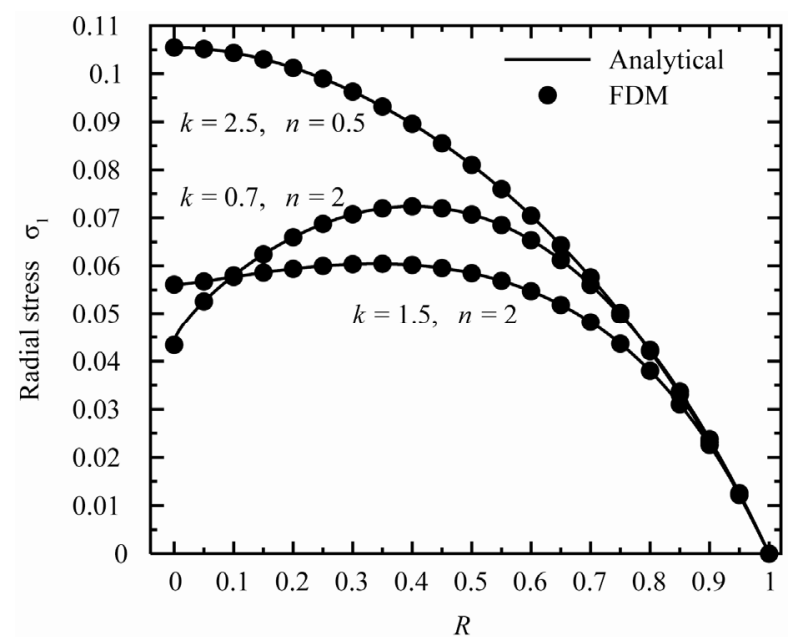

Figure 4. Radial stress $\sigma_{1}$ of the variable-thickness solid disk for different values of $k$ and $n$.

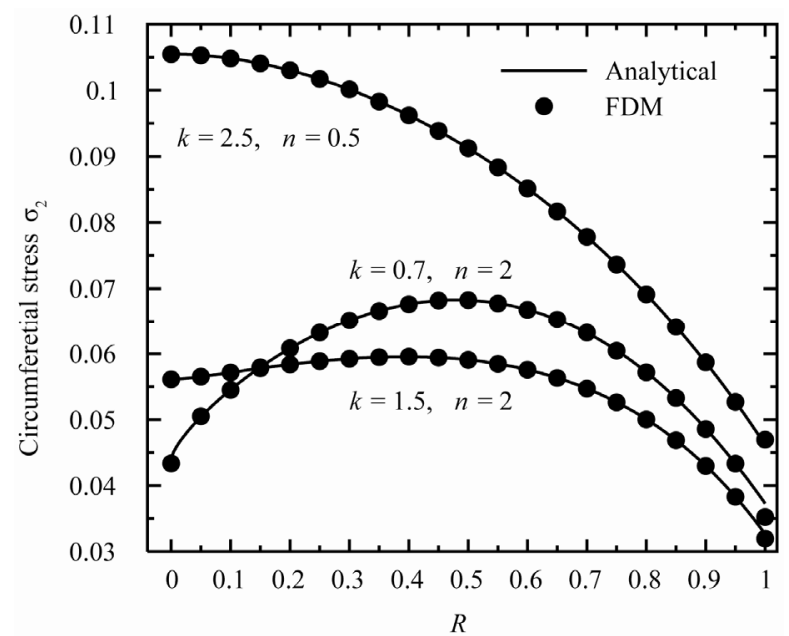

Figure 5. Circumferential stress $\sigma_{2}$ of the variable-thick- ness solid disk for different values of $k$ and $n$.

consequently, stresses with excellent accuracy with the exact analytical solution. In most cases of rotating variable-thickness solid disks, the analytical solutions are not available. In these cases, one can trustily use the present FDM solutions.

\section{CONCLUSIONS}

The rotating solid disk with variable thickness is treated herein. By introducing a suitable stress function, the governing equation is derived from the equation of motion of rotating disk, compatibility equation and the proposed stress-strain relationship. Both the analytical and numerical solutions are presented. The calculation of the rotating solid disk is turned into finding the solution of a second-order differential equation under the given conditions at the center and the outer edge of the disk. The numerical solution is based upon the finite difference method. The governing equation is solved analytically with the help of Whittaker's functions and a number of numerical examples are studied. The results of the two solutions at different disk configurations are compared. The proposed FDM approach gives very agreeable results to the analytical solution and so it may be used for different problems that analytical solutions are not available.

\section{REFERENCES}

[1] Timoshenko, S.P. and Goodier, J.N. (1970) Theory of elasticity. McGraw-Hill, New York.

[2] Ugural, S.C. and Fenster, S.K. (1987) Advanced strength and applied elasticity. Elsevier, New York.

[3] Gamer, U. (1984) Elastic-plastic deformation of the rotating solid disk. Ingenieur-Archiv, 54, 345-354. doi:10.1007/BF00532817

[4] Gamer, U. (1985) Stress distribution in the rotating elas- 
tic-plastic disk. ZAMM, 65, T136-137.

[5] Eraslan, A.N. (2000) Inelastic deformation of rotating variable thickness solid disks by Tresca and Von Mises criteria. International Journal of Computational Engineering Science, 3, 89-101.

doi:10.1142/S1465876302000563

[6] Eraslan, A.N. and Orcan, Y. (2002) On the rotating elastic-plastic solid disks of variable thickness having concave profiles. International Journal of Mechanical Sciences, 44, 1445-1466.

doi:10.1016/S0020-7403(02)00038-3

[7] Eraslan, A.N. (2005) Stress distributions in elastic-plastic rotating disks with elliptical thickness profiles using Tresca and von Mises criteria. ZAAM, 85, 252-266.

[8] Zenkour, A.M. and Allam, M.N.M. (2006) On the rotating fiber-reinforced viscoelastic composite solid and annular disks of variable thickness. International Journal for Computational Methods in Engineering Science, 7, 21-31. doi:10.1080/155022891009639

[9] Zienkiewicz, O.C. (1971) The finite element method in engineering science. McGraw-Hill, London.

[10] Banerjee, P.K. and Butterfield, R. (1981) Boundary element methods in engineering science. McGraw-Hill, New York.

[11] You, L.H., Tang, Y.Y., Zhang, J.J. and Zheng, C.Y. (2000) Numerical analysis of with elastic-plastic rotating disks arbitrary variable thickness and density. The International Journal of Solids and Structures, 37, 7809-7820. doi:10.1016/S0020-7683(99)00308-X

[12] Zenkour, A.M. and Mashat, D.S. (2010), Analytical and numerical solutions for a rotating disk of variable thickness. Applied Mathematics, 1, 430-437. doi:10.4236/am.2010.15057 